\title{
The Journey to Advanced Clinical Pharmacy \\ Practice: Global Collaboration Will \\ Accelerate the Pace
}

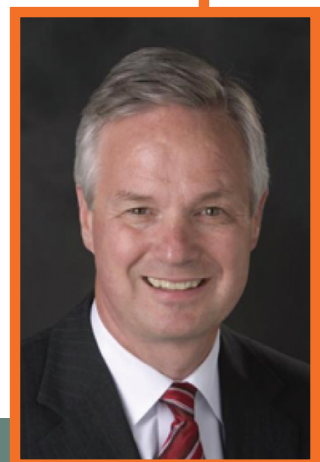

\author{
Paul W. Bush \\ PharmD, MBA, BCPS, FASHP, \\ Vice President, Global Resource Development and Consulting - ASHP \\ Email: pbush@ashp.org
}

Paul Bush is the Vice President for Global Resource Development and Consulting for the American Society of Health-System Pharmacists (ASHP). He has held the positions of Chief Pharmacy Officer for Duke University Hospital in Durham, NC, Director of Pharmacy Services for Medical University of South Carolina, St. John Hospital and Medical Center and Detroit Osteopathic Hospital and was Corporate Director for Clinical Pharmacy Services for Horizon Health System.

Dr. Bush received his B.S. in Pharmacy from the University of Michigan and Pharm.D. and M.B.A. from Wayne State University in Detroit, Michigan. He serves on the faculty of University of North Carolina Eschelman School of Pharmacy and Campbell University College of Pharmacy and Health Sciences. He previously served on the faculty of the Medical University of South Carolina (19992009) and Wayne State University (19841999) and served as Clinical Associate Dean for Medical Center and Health Systems for the South Carolina College of Pharmacy.

He has served as presidential officer, board member and Chair of the Board of Directors of ASHP. Dr. Bush is Past-Treasurer of the Michigan Pharmacists Association. He has served on the Michigan Society of Health System Pharmacists Board of Directors and held the position of President-elect.

$\mathrm{He}$ co-authored Managing and Leading 44 Lessons Learned for Pharmacists, and chapters in Building a Successful Ambulatory Practice, Financial Management Basics for Health System Pharmacists, Handbook of Institutional Pharmacy Practice, and the Pharmacy Certified Technician Training Manual.

Dr. Bush was awarded the John W. Webb Lecture Award by Northeastern University and ASHP, an honorary Doctor of Science degree from Campbell University, the Distinguished Alumnus Award from the Wayne State University Pharmacy Alumni Association, the Dean Golod Award from MUSC Health, the Distinguished Service Award by the ASHP Section of Pharmacy Practice Managers and the NCAP Pharmacy Ambassador Award
The Arab civilization's early contribution to pharmacy was recognized in the late 700's when pharmacy attained a professional identity and independent pharmacy practice was established through the introduction of the pharmacy shop. ${ }^{[1]}$ In the ninth century pharmaceutical educational was formalized in the Arab world and pharmacy achieved recognition as a profession. [1]

The pharmacist was responsible for preparing and dispensing medications based on prescriptions enhancing the safety of the medication use process. Pharmacy practice in the Arab world has continuously evolved and today there are many centers with progressive pharmacy programs with comprehensive clinical services. As an example of progress, in 2008, a clinical pharmacy program became part of pharmaceutical education in Egypt and pharmacy schools began to prepare pharmacists for clinical practice. ${ }^{[2]}$

The movement from a product orientation to a patient focused clinical role has progressed at varying paces around the globe. The model was initially described as clinical pharmacy - defined as the branch of pharmacy that involves the provision of patient care with the use of medications to optimize the health outcomes of patients. [3] In the late 1990's the term pharmaceutical care was popular and today, the model is often referred to as medication management.

The journey from a product preparation and dispensing orientation to a patient focused clinical role has occurred in the United States during my 40-year professional career. While a pharmacy student at the University of Michigan in the mid-1970s I gained practice experience at a community hospital on weekends as a pharmacy intern. Services were limited to review of new medication orders, preparation, dispensing, and provision of drug information when requested. We introduced our first clinical service, a consultbased aminoglycoside pharmacokinetic dosing service in 1980 and deployed our first clinical pharmacist to an acute care patient unit to "provide convenient and specialized pharmacy services". The pharmacist participated with the care team, provided drug information, patient drug-profile monitoring, intravenous therapy consultation, code team response, medication order review and first-dose dispensing. This is just one example of the movement toward clinical practice underway in the US at the time.

The vision of pharmacy leaders was for the pharmacy profession to provide efficient, effective, and safe medication accessibility with extensive deployment of clinically trained pharmacists working collaboratively with physicians, nurses and other clinicians to improve patient outcomes. To create momentum several consensus conferences were convened. In 1985, Directions for Clinical Practice 
in Pharmacy - Hilton Head Conference was convened to assess the current state of clinical practice of pharmacy, set goals and identify practical ways to advance clinical practice. Then four years later the Pharmacy in the 21st Century Conference was held. Participants examined major issues that would confront the profession in the ensuing 15-20 years and identified strategies to address opportunities and responsibilities in pharmaceutical care. The term pharmaceutical care became widely used in the 1990's and was viewed to encompass both the clinical role of the pharmacist, as well as other activities of pharmacists, including medication preparation and dispensing.

It was defined as the direct, responsible provision of medication-related care for the purpose of achieving definite outcomes that improve a patient's quality of life. ${ }^{[4]}$ In 1993, more than 200 participants met in San Antonio at the ASHP Conference on Implementing Pharmaceutical Care. Participants identified critical skills needed to provide pharmaceutical care, the need for a departmental strategic plan, personal commitment of the entire pharmacy staff, and support of boards of pharmacy to enable pharmacists to provide pharmaceutical care. To support the movement, schools of pharmacy adopted the Doctor of Pharmacy curriculum, and the hospitals dramatically increased the number of post-graduate residency programs.

Significant practice changed was underway, but it became clear the "practice model" utilized in health system pharmacy would need to change for pharmacists to realize their full potential in providing direct patient care. In 2010, ASHP convened the Pharmacy Practice Model Initiative (PPMI) Summit to create passion, commitment, and action among hospital and healthsystem pharmacy practice leaders to significantly advance the health and well-being of patients. The objectives of the PPMI were to create a framework for the practice model, determine services, identify emerging technologies, develop a template, and identify specific actions pharmacy leaders and staff should take to implement practice model change. The PPMI stimulated the action and change needed. Many departments transitioned to an integrated practice model, pharmacists enhanced their clinical capabilities, the pharmacy technician's role expanded, and information and automation technology was adopted.

The journey continues in the US. Most health systems have adopted an integrated practice model with pharmacists deployed to patient care units or assigned to teams. Today's focus is further deployment of clinical pharmacists to additional patient care units and ambulatory clinics. The leadership and support provided by professional societies has been key to successful progress. The ASHP Practice Advancement Initiative (PAI) 2030 provides momentum and a path forward with 59 recommendations on providing optimal, safe, and effective medication use, aspirational guidance serving as a roadmap to pharmacy advancement and a future-focused set of concepts looking beyond today's barriers to change. PAI 2030 themes for practice change focus on optimizing care via pharmacist-provided comprehensive medication management, integrating the pharmacy enterprise for convenient and costeffective care, advancing pharmacy technician roles, and adopting personalized, targeted therapies. [5]

Globally, pharmacy practice is evolving to patient focused interprofessional team-based care. The pace of change varies from region to region, but the vision of pharmacy as a clinical profession is constant. What is different today and has been a game-changer is the robust capability of willing and engaged pharmacists across the globe to communicate, share strategies and collaborate using information technology. Social media, video calls and web-based meeting capability has dramatically improved global communication and collaboration.

I have shared the journey in the US to illustrate that practice change takes time and coordinated effort. What I have described is not unique to the US and has been occurring in countries around the world. The pace of change varies from one country to the next for unique but important reasons. Pharmacy leaders across the globe can enhance the pace of change by working together to identify barriers, design solutions, and implement change.

\section{References}

[01] D. L. Cowen and W. H. Helfand, Pharmacy. an illustrated history. New York : Harry N. Abrams, Inc, 1990.

[02] M. M. Abdel-Latif and K. Sabra, "Clinical pharmacy practice in Egyptian hospitals." American Journal of Hospital Pharmacy, Vol. 73, pp. 63-66, 2016.

[03] "American College of Clinical Pharmacy. The definition of clinical pharmacy." Pharmacotherapy; Vol. 28, no. 6, pp. 816-817, 2008.

[04] "American Society of Hospital Pharmacists. ASHP statement on pharmaceutical care." American Journal of Hospital Pharmacy, Vol. 50, pp. 1720-1723, 1993.

[05] "ASHP Practice Advancement Initiative 2030: New recommendations for advancing pharmacy practice in health systems." American Journal of Health-System Pharmacy, Vol. 77, pp. 113- 121, 2020. 